\title{
Information Fusion Filter for Multi-rate Multi-sensor Systems
}

\author{
Jing $\mathrm{Ma}^{1}$, Hao $\mathrm{Jin}^{2}$, Tian Tian $^{3}$ and Wei Zhang ${ }^{1}$ \\ ${ }^{1}$ School of Mathematics Science, Heilongjiang University, Harbin 150080, China \\ ${ }^{2}$ School of Computer and Information Engineering, Harbin University of \\ Commerce, Harbin 150028, China \\ ${ }^{3}$ Department of Foreign Languages Teaching and Research, Heilongjiang \\ University, Harbin 150080, China \\ Imajing427@gmail.com
}

\begin{abstract}
This paper is concerned with the information fusion filtering problem for a class of multi-rate multi-sensor systems, where the system is described at the highest sampling rate and different sensors may have different lower sampling rates. Firstly, the local filters (LFs) at state updating points are proposed by using the LFs at measurement sampling points. Then, the distributed suboptimal fusion filter is obtained by the wellknown covariance intersection fusion (CIF) algorithm. The filtering error variance matrices are derived to obtain the fusion weights. The computational cost is reduced since the cross-covariance matrices between any two local filters are avoided. Simulation example verifies the correctness and feasibility of the proposed algorithm.
\end{abstract}

Keywords: Multi-rate; Multi-sensor; Covariance Intersection Fusion; Filter

\section{Introduction}

In recent years, the information fusion filtering problem for systems with multiple sensors has gained lots of attention due to the widely applications such as target tracking, single processing and robot navigation [1].

When the stochastic system is measured by multiple sensors, there are two approaches to process the multiple measurements from different sensors. One is the centralized filter, the other is distributed fusion filter [2]. The centralized filter can give the global optimal estimation. However, it can result in high computational cost due to the high dimension augmented measurement. Recently, many researchers are focus on the distributed filter since it is easily for fault detection and isolation. There are many popular distributed fusion algorithms such as federated square-root filter [3], maximum likelihood fusion algorithm [4], weighting fusion algorithms in the linear minimum variance sense [5] and CIF algorithm [6]. However, the above algorithms are only suitable for single rate systems.

For multi-rate systems, the first important study goes back to the switch decomposition technique proposed by Kranc [7]. Generally, there are two methods for the state estimation problem for multi-rate systems. One is based on multiscale system theory and the other is based on Kalman filtering theory. On the basis of multiscale system theory, many famous fusion strategies are proposed for multirate systems with the sampling rate ratio being one or positive integer power to two. However, the state estimators are very complex and high computational burden. On the basis of Kalman filtering theory, many useful filtering strategies are proposed such as optimal signal reconstruction method [8], asynchronous centralized fusion algorithm [9], sequential filtering algorithm [10], left synchronously lifting technology [11], and measurement augmented approach [12]. But, the computational cost of the above filtering strategies is high since they are given by 
state/measurement augmentation. In order to avoid the state augmentation, the multi-rate fusion problem is transformed into an equivalent single rate fusion problem. For non-uniform sampling systems, a distributed fusion filter is given [13], for uniform sampling systems, the corresponding distributed fusion filters are also given in [14-15]. However, the cross-covariance matrices are needed to obtain the fusion weights. Furthermore, the multi-rate fusion filters for systems with network constrains are studied in [16-18]. However, the state/measurement augmentation is not avoided.

In this article, a new LF is obtained by using the LF for the measurement sampling points. Then, the well-known CIF algorithm is used to fuse all the LFs. The proposed new LF can reduce the computational cost since the state augmentation is avoided. The proposed CIF filter can further reduce the computational burden since the cross-covariance matrices are avoided.

\section{Problem Formulation}

Consider the following linear discrete-time stochastic multi-rate systems measured by $Q$ sensors

$$
\begin{gathered}
x(t b+b)=\Phi x(t b)+\Gamma w(t b) \\
z_{r}\left(t b_{r}\right)=H_{r} x\left(t b_{r}\right)+v_{r}\left(t b_{r}\right), b_{r}=c_{r} b, r \in\{1, \cdots, Q\}
\end{gathered}
$$

where $x(t b) \in \square$ " is the state vector, $z_{r}\left(t b_{r}\right) \in \square^{n_{r}}, r \in\{1, \cdots, Q\}$ are the measured outputs, $\Phi$, $\Gamma$ and $H_{r}, r \in\{1, \cdots, Q\}$ are the constant matrices with suitable dimensions. $w(t b) \in \square^{q}$ and $v_{r}\left(t b_{r}\right) \in \square n_{r}, r \in\{1, \cdots, Q\}$ are white noises. The state $x(t b)$ is updated at the highest rate with a period $b$ and the $r$ th sensor measurement $z_{r}\left(t b_{r}\right) \in{ }^{n_{r}}$ is sampled at a lower rate with a period $b_{r}=c_{r} b$ where $c_{r}$ is a positive integer. Superscript $r$ stands for the $r$ th sensor.

Assumption $1 w(t b)$ and $v_{r}\left(t b_{r}\right)$ are uncorrelated white noises with zero means and variance matrices $R^{w}$ and $R_{r}^{v}$, respectively.

Assumption 2 The initial state vector $x(0)$ is uncorrelated with $w(t b)$ and $v_{r}\left(t b_{r}\right)$, and satisfies

$$
\mathrm{E}\{x(0)\}=\sigma \text { and } \mathrm{E}\left\{(x(0)-\sigma)(x(0)-\sigma)^{\mathrm{T}}\right\}=\Omega
$$

Our objective is to find the distributed CIF filter $\hat{x}_{o}(t b)$ based on the measurement information $\left(z_{r}\left(t b_{r}\right), z_{r}\left(t b_{r}-b_{r}\right), \cdots, z_{r}(0)\right), r \in\{1, \cdots, Q\}$.

\section{Distributed Information Filter}

\subsection{Local Filter}

In this subsection, we first establish the state space model at the measurement sampling points of the $r$ th sensor[2].

$$
\begin{gathered}
x\left(t b_{r}+b_{r}\right)=\Phi_{r} x\left(t b_{r}\right)+\Gamma_{r} w_{r}\left(t b_{r}\right) \\
z_{r}\left(t b_{r}\right)=H_{r} x\left(t b_{r}\right)+v_{r}\left(t b_{r}\right)
\end{gathered}
$$

where

$\Phi_{r}=\Phi^{c_{r}}, \Gamma_{r}=\left[\begin{array}{llllll}\Phi^{c_{r}-1} \Gamma & \Phi^{c_{r}-2} \Gamma & \cdots & \Gamma\end{array}\right], w_{r}\left(t b_{r}\right)=\left[\begin{array}{llll}w^{\mathrm{T}}\left(t b_{r}\right) & w^{\mathrm{T}}\left(t b_{r}+b\right) & \cdots & w^{\mathrm{T}}\left(t b_{r}+b_{r}-b\right)\end{array}\right]^{\mathrm{T}}$.

Further we have the following statistical property

$$
\mathrm{E}\left[w_{r}\left(t b_{r}\right)\right]=0, R_{r}^{w}=\mathrm{E}\left[w_{r}\left(t b_{r}\right) w_{r}^{\mathrm{T}}\left(t b_{r}\right)\right]=\operatorname{diag}\left(R^{w}, \cdots, R^{w}\right)_{c_{r} q \times c_{r} q}
$$

Observe that systems (4) and (5) are transformed into the single rate systems. In the following, we will give the LFs $\underline{\hat{x}}_{r}\left(t b_{r} \mid t b_{r}\right)$ and the corresponding estimation error 
variance matrices $\underline{P}_{r}\left(t b_{r} \mid t b_{r}\right)$ at the measurement sampling points for the $r$ th sensor subsystem by applying the classical Kalman filter.

Lemma 1[2] Under Assumptions 1 and 2, the LFs for systems (4) and (5) are computed by

$$
\begin{gathered}
\underline{\hat{x}}_{r}\left(t b_{r} \mid t b_{r}\right)=\underline{\hat{x}}_{r}\left(t b_{r} \mid t b_{r}-b_{r}\right)+K_{r}\left(t b_{r}\right) \underline{\varepsilon}_{r}\left(t b_{r}\right) \\
\underline{\hat{x}}_{r}\left(t b_{r}+b_{r} \mid t b_{r}\right)=\Phi_{r} \underline{\hat{x}}_{r}\left(t b_{r} \mid t b_{r}-b_{r}\right)+L_{r}\left(t b_{r}\right) \underline{\varepsilon}_{r}\left(t b_{r}\right) \\
\underline{\varepsilon}_{r}\left(t b_{r}\right)=z_{r}\left(t b_{r}\right)-H_{r} \underline{\hat{x}}_{r}\left(t b_{r} \mid t b_{r}-b_{r}\right) \\
K_{r}\left(t b_{r}\right)=\underline{P}_{r}\left(t b_{r} \mid t b_{r}-b_{r}\right) H_{r}^{\mathrm{T}} \underline{Q}_{s_{r}}^{-1}\left(t b_{r}\right) \\
L_{r}\left(t b_{r}\right)=\Phi_{r} K_{r}\left(t b_{r}\right) \\
\underline{Q}_{s_{r}}\left(t b_{r}\right)=H_{r} \underline{P}_{r}\left(t b_{r} \mid t b_{r}-b_{r}\right) H_{r}^{\mathrm{T}}+R_{r}^{v} \\
\underline{P}_{r}\left(t b_{r} \mid t b_{r}\right)=\underline{P}_{r}\left(t b_{r} \mid t b_{r}-b_{r}\right)-K_{r}\left(t b_{r}\right) \underline{Q}_{\varepsilon_{r}}\left(t b_{r}\right) K_{r}^{\mathrm{T}}\left(t b_{r}\right) \\
\left.+\underline{P}_{r}\right)=\left(\Phi_{r}-L_{r}\left(t b_{r}\right) H_{r}\right) \underline{P}_{r}\left(t b_{r} \mid t b_{r}-b_{r}\right)\left(\Phi_{r}-L_{r}\left(t b_{r}\right) H_{r}\right)^{\mathrm{T}} \\
+\Gamma_{r} R_{r}^{w} \Gamma_{r}{ }^{\mathrm{T}}+L_{r}\left(t b_{r}\right) R_{r}^{v} L_{r}^{\mathrm{T}}\left(t b_{r}\right)
\end{gathered}
$$

where $\underline{\hat{x}}_{r}\left(t b_{r} \mid t b_{r}\right)$ is the filter, $\underline{\hat{x}}_{(}\left(t b_{r} \mid t b_{r}-b_{r}\right)$ is the one-step predictor at the measurement sampling points, $\underline{\varepsilon}_{r}\left(t b_{r}\right)$ is the innovation sequence with variance $Q_{\varepsilon_{r}}\left(t b_{r}\right), K_{r}\left(t b_{r}\right)$ is the filtering gain, $L_{r}\left(t b_{r}\right)$ is the one-step prediction gain, $\underline{P}_{(}\left(t b_{r} \mid t b_{r}\right)$ is the filtering error variance matrix, $\underline{P}_{r}\left(t b_{r} \mid t b_{r}-b_{r}\right)$ is the one-step prediction error variance matrix. The initial values are $\underline{\hat{x}}_{r}(0 \mid-1)=\sigma$ and $\underline{P}_{r}(0 \mid-1)=\Omega$.

Next, we will derive the LFs $\hat{x}_{r}\left(t b_{r}-l b \mid t b_{r}\right), l \in\left\{1, \cdots, c_{r}\right\}$ at the state updating points by applying the LFs $\underline{\hat{x}}_{r}\left(t b_{r} \mid t b_{r}\right)$ at the measurement sampling points and the state update equation.

Theorem 1 Under Assumptions 1 and 2, for systems (1) and (2), we have the LFs $\hat{x}_{r}\left(t b_{r}-l b \mid t b_{r}\right), l=1,2, \cdots, c_{r}$ at the state updating points as follows

$$
\begin{gathered}
\hat{x}_{r}\left(t b_{r}-l b \mid t b_{r}\right)=\Phi^{-l}\left[\underline{\hat{x}}_{r}\left(t b_{r} \mid t b_{r}\right)-\sum_{k=1}^{l} \Phi^{k-1} \Gamma \hat{w}_{r}\left(t b_{r}-k b \mid t b_{r}\right)\right] \\
\hat{w}_{r}\left(t b_{r}-k b \mid t b_{r}\right)=M_{r}\left(t b_{r}-k b \mid t b_{r}\right) \underline{\varepsilon}_{r}\left(t b_{r}\right), k \in\{1, \cdots, l\} \\
M_{r}\left(t b_{r}-k b \mid t b_{r}\right)=R^{w} \Gamma^{\mathrm{T}}\left(\Phi^{k-1}\right)^{\mathrm{T}} H_{r}^{\mathrm{T}} \underline{Q}_{\varepsilon_{r}}^{-1}\left(t b_{r}\right), k \in\{1, \cdots, l\}
\end{gathered}
$$

where $\underline{\varepsilon}_{r}\left(t b_{r}\right), \underline{Q}_{\varepsilon_{r}}\left(t b_{r}\right), \underline{\hat{\hat{x}}}_{r}\left(t b_{r} \mid t b_{r}\right)$ are given by Lemma 1 .

Proof: From the iteration of (1)

$$
x\left(t b_{r}\right)=\Phi^{l} x\left(t b_{r}-l b\right)+\sum_{k=1}^{l} \Phi^{k-1} \Gamma w\left(t b_{r}-k b\right), l \in\left\{1, \cdots, c_{r}\right\}
$$

By arranging (18)

$$
x\left(t b_{r}-l b\right)=\Phi^{-l}\left[x\left(t b_{r}\right)-\sum_{k=1}^{l} \Phi^{k-1} \Gamma w\left(t b_{r}-k b\right)\right]
$$

Taking projection of both sides of (19) on the linear space $L\left(z_{r}\left(t b_{r}\right), z_{r}\left(t b_{r}-b_{r}\right), \cdots, z_{r}(0)\right)$, we have (15). The white noise smoother $\hat{w}_{r}\left(t b_{r}-k b \mid t b_{r}\right)$ is obtained by the following recursive projection equation

$$
\hat{w}_{r}\left(t b_{r}-k b \mid t b_{r}\right)=\hat{w}_{r}\left(t b_{r}-k b \mid t b_{r}-b_{r}\right)+M_{r}\left(t b_{r}-k b \mid t b_{r}\right) \underline{\varepsilon}_{r}\left(t b_{r}\right), k \in\{1, \cdots, l\}
$$

From the uncorrelation of $w\left(t b_{r}-k b\right)$ and $L\left(z_{r}\left(t b_{r}-b_{r}\right), \cdots, z_{r}(0)\right)$, we have the white noise predictors

$$
\hat{w}_{r}\left(t b_{r}-k b \mid t b_{r}-b_{r}\right)=0, k \in\{1, \cdots, l\}
$$


Substituting (21) into (20), we can obtain (16). The white noise smoothing gain matrices $M_{r}\left(t b_{r}-k b \mid t b_{r}\right)$ are given by

$$
M_{r}\left(t b_{r}-k b \mid t b_{r}\right)=\mathrm{E}\left[w\left(t b_{r}-k b\right) \underline{\varepsilon}_{r}^{\mathrm{T}}\left(t b_{r}\right)\right] \underline{Q}_{\varepsilon_{r}}^{-1}\left(t b_{r}\right)
$$

Substituting (2) into (9), the innovation sequences can be rewritten as

$$
\underline{\varepsilon}\left(t b_{r}\right)=H_{r} \underline{\tilde{x}}_{(}\left(t b_{r} \mid t b_{r}-b_{r}\right)+v_{r}\left(t b_{r}\right)
$$

From $\underline{\tilde{x}}_{r}\left(t b_{r} \mid t b_{r}-b_{r}\right)=x\left(t b_{r}\right)-\underline{\hat{x}}_{r}\left(t b_{r} \mid t b_{r}-b_{r}\right), \quad w\left(t b_{r}-k b\right) \perp \underline{\hat{x}}_{r}\left(t b_{r} \mid t b_{r}-b_{r}\right) \quad, \quad$ (18) and Assumption 1, we have

$$
\mathrm{E}\left[w\left(t b_{r}-k b\right) \underline{\varepsilon}^{\mathrm{T}}\left(t b_{r}\right)\right]=\mathrm{E}\left[w\left(t b_{r}-k b\right) x^{\mathrm{T}}\left(t b_{r}\right)\right] H_{r}^{\mathrm{T}}=R^{\mathrm{w}} \Gamma^{\mathrm{T}}\left(\Phi^{k-1}\right)^{\mathrm{T}} H_{r}^{\mathrm{T}}
$$

Substituting (24) into (22), (17) is obtained.

Remark 1: From Theorem 1, we see that the LFs at the state updating points are derived by applying the LFs at the measurement sampling points. Therefore, compared with the state augmented approach, the proposed filter can obviously reduce the computational burden for the $r$ th sensor subsystem. Now, we give the computational cost at each measurement sampling points: our filter is $\mathrm{O}\left(n^{3}\right)$ and the augmented filter in [12] is $\mathrm{O}\left(\left(c_{r} n\right)^{3}\right)$. On the other hand, from the point of view of using information, the two filters have the same estimation accuracy. We verify it in the simulation research.

Now, we have obtained the LFs at the state updating points. In the following text, we need to derive the filtering error variance matrices to obtain the fusion weights.

\subsection{Fusion Filter}

Theorem 2 Under Assumptions 1 and 2, for systems (1) and (2), we have the local filtering error variance matrices at the state updating points $P_{r}\left(t b_{r}-l b \mid t b_{r}\right), l \in\left\{1, \cdots, c_{r}\right\}$ as follows

$$
\begin{gathered}
P_{r}\left(t b_{r}-l b \mid t b_{r}\right)=\Phi^{-l} P_{r}\left(t b_{r} \mid t b_{r}\right)\left(\Phi^{-l}\right)^{\mathrm{T}} \\
+\sum_{k, m=1}^{\prime} \Phi^{k-1-l} \Gamma P_{r}^{w}\left(t b_{r}-k b, t b_{r}-m b \mid t b_{r}\right) \Gamma^{\mathrm{T}}\left(\Phi^{m-1-l}\right)^{\mathrm{T}} \\
-\sum_{m=1}^{l} \Phi^{-l} P_{r}^{x w}\left(t b_{r}, t b_{r}-m b \mid t b_{r}\right) \Gamma^{\mathrm{T}}\left(\Phi^{m-1-l}\right)^{\mathrm{T}}-\sum_{k=1}^{l} \Phi^{k-1-1} \Gamma P_{r}^{w x}\left(t b_{r}-k b, t b_{r} \mid t b_{r}\right)\left(\Phi^{-l}\right)^{\mathrm{T}} \\
P_{r}^{w}\left(t b_{r}-k b, t b_{r}-m b \mid t b_{r}\right)=R^{w} \delta_{k m}-R^{w} \Gamma^{\mathrm{T}}\left(\Phi^{k-1}\right)^{\mathrm{T}} H_{r}^{\mathrm{T}} M_{r}^{\mathrm{T}}\left(t b_{r}-m b \mid t b_{r}\right) \\
-M_{r}\left(t b_{r}-k b \mid t b_{r}\right) H_{r} \Phi^{m-1} \Gamma R^{w}+M_{r}\left(t b_{r}-k b \mid t b_{r}\right) H_{r} P_{r}\left(t b_{r} \mid t b_{r}-b_{r}\right) H_{r}^{\mathrm{T}} M_{r}^{\mathrm{T}}\left(t b_{r}-m b \mid t b_{r}\right) \\
+M_{r}\left(t b_{r}-k b \mid t b_{r}\right) R_{r}^{v} M_{r}^{\mathrm{T}}\left(t b_{r}-m b \mid t b_{r}\right), k, m \in\{1, \cdots, l\} \\
P_{r}^{x^{w}}\left(t b_{r}, t b_{r}-k b \mid t b_{r}\right)=\left(I_{n}-K_{r}\left(t b_{r}\right) H_{r}\right) \Phi^{k-1} \Gamma R^{w}, k \in\{1, \cdots, l\}
\end{gathered}
$$

where $\delta_{k m}$ is Kronecker delta function, $P_{r}^{w}\left(t b_{r}-k b, t b_{r}-m b \mid t b_{r}\right)$ is the white noise smoothing error variance matrix, $P_{r}^{x^{w}}\left(t b_{r}, t b_{r}-k b \mid t b_{r}\right)$ is the covariance matrix between state and system noise, also we have $P_{r}^{w x}\left(t b_{r}-k b, t b_{r} \mid t b_{r}\right)=\left(P_{r}^{x w}\left(t b_{r}, t b_{r}-k b \mid t b_{r}\right)\right)^{\mathrm{T}}$. The initial values are $\underline{P}_{r}\left(t b_{r} \mid t b_{r}\right)$ and $\underline{P}_{(}\left(t b_{r} \mid t b_{r}-b_{r}\right)$ computed by Lemma 1 .

Proof: Subtracting (15) from $x\left(t b_{r}-l b\right)$, we have the estimation error variance equation

$$
\begin{gathered}
\tilde{x}_{r}\left(t b_{r}-l b \mid t b_{r}\right)=x\left(t b_{r}-l b\right)-\hat{x}_{r}\left(t b_{r}-l b \mid t b_{r}\right) \\
=x\left(t b_{r}-l b\right)-\Phi^{-l}\left[\underline{\hat{x}}_{(}\left(t b_{r} \mid t b_{r}\right)-\sum_{k=1}^{l} \Phi^{k-1} \Gamma \hat{w}_{r}\left(t b_{r}-k b \mid t b_{r}\right)\right] \\
=\Phi^{-1}\left[x\left(t b_{r}\right)-\sum_{k=1}^{l} \Phi^{k-1} \Gamma w\left(t b_{r}-k b\right)\right]-\Phi^{-l}\left[\underline{\hat{x}}_{r}\left(t b_{r} \mid t b_{r}\right)-\sum_{k=1}^{l} \Phi^{k-1} \Gamma \hat{w}_{r}\left(t b_{r}-k b \mid t b_{r}\right)\right]
\end{gathered}
$$




$$
=\Phi^{-l} \underline{\tilde{x}}_{r}\left(t b_{r} \mid t b_{r}\right)-\sum_{k=1}^{l} \Phi^{k-1-l} \Gamma \tilde{w}_{r}\left(t b_{r}-k b \mid t b_{r}\right)
$$

From(28), we have the estimation error variance matrix at the state updating points

$$
\begin{gathered}
P_{r}\left(t b_{r}-l b \mid t b_{r}\right)=\mathrm{E}\left[\tilde{x}_{r}\left(t b_{r}-l b \mid t b_{r}\right) \tilde{x}_{r}^{\mathrm{T}}\left(t b_{r}-l b \mid t b_{r}\right)\right] \\
=\Phi^{-l} \underline{P}_{r}\left(t b_{r} \mid t b_{r}\right)\left(\Phi^{-l}\right)^{\mathrm{T}}+\sum_{k, m=1}^{l} \Phi^{k-1-l} \Gamma \mathrm{E}\left[\tilde{w}_{r}\left(t b_{r}-k b \mid t b_{r}\right) \tilde{w}_{r}^{\mathrm{T}}\left(t b_{r}-m b \mid t b_{r}\right)\right] \Gamma^{\mathrm{T}}\left(\Phi^{m-1-l}\right)^{\mathrm{T}} \\
-\sum_{m=1}^{l} \Phi^{-l} \mathrm{E}\left[\tilde{x}_{r}\left(t b_{r} \mid t b_{r}\right) \tilde{w}_{r}^{\mathrm{T}}\left(t b_{r}-m b \mid t b_{r}\right)\right] \Gamma^{\mathrm{T}}\left(\Phi^{m-1-l}\right)^{\mathrm{T}} \\
-\sum_{k=1}^{l} \Phi^{k-1-l} \Gamma \mathrm{E}\left[\tilde{w}_{r}\left(t b_{r}-k b \mid t b_{r}\right) \underline{\tilde{x}}_{r}^{\mathrm{T}}\left(t b_{r} \mid t b_{r}\right)\right]\left(\Phi^{-l}\right)^{\mathrm{T}}
\end{gathered}
$$

Subtracting (16) from $w\left(t b_{r}-k b\right)$, and using (23), we have the white noise estimation error equation

$$
\begin{gathered}
\tilde{w}_{r}\left(t b_{r}-k b \mid t b_{r}\right)=w\left(t b_{r}-k b\right)-\hat{w}_{r}\left(t b_{r}-k b \mid t b_{r}\right) \\
=w\left(t b_{r}-k b\right)-M_{r}\left(t b_{r}-k b \mid t b_{r}\right) H_{r} \underline{\tilde{x}}_{r}\left(t b_{r} \mid t b_{r}-b_{r}\right)-M_{r}\left(t b_{r}-k b \mid t b_{r}\right) v_{r}\left(t b_{r}\right)
\end{gathered}
$$

From (30), the uncorrelation of $\underline{\tilde{x}}_{r}\left(t b_{r} \mid t b_{r}-b_{r}\right)$ and $v_{r}\left(t b_{r}\right)$, and Assumption 1, the white noise smoothing error variance matrix is computed by

$$
\begin{aligned}
& P_{r}^{w}\left(t b_{r}-k b, t b_{r}-m b \mid t b_{r}\right)=\mathrm{E}\left[\tilde{w}_{r}\left(t b_{r}-k b \mid t b_{r}\right) \tilde{w}_{r}^{\mathrm{T}}\left(t b_{r}-m b \mid t b_{r}\right)\right], k, m \in\{1, \cdots, l\} \\
& =\mathrm{E}\left[w\left(t b_{r}-k b\right) w^{\mathrm{T}}\left(t b_{r}-m b\right)\right]-\mathrm{E}\left[w\left(t b_{r}-k b\right) \underline{\tilde{x}}_{r}^{\mathrm{T}}\left(t b_{r} \mid t b_{r}-b_{r}\right)\right] H_{r}^{\mathrm{T}} M_{r}^{\mathrm{T}}\left(t b_{r}-m b \mid t b_{r}\right) \\
& \quad-M_{r}\left(t b_{r}-k b \mid t b_{r}\right) H_{r} \mathrm{E}\left[\tilde{\tilde{x}}_{r}\left(t b_{r} \mid t b_{r}-b_{r}\right) w^{\mathrm{T}}\left(t b_{r}-m b\right)\right] \\
& +M_{r}\left(t b_{r}-k b \mid t b_{r}\right) H_{r} \mathrm{E}\left[\tilde{x}_{r}\left(t b_{r} \mid t b_{r}-b_{r}\right) \tilde{\tilde{x}}_{r}^{\mathrm{T}}\left(t b_{r} \mid t b_{r}-b_{r}\right)\right] H_{r}^{\mathrm{T}} M_{r}^{\mathrm{T}}\left(t b_{r}-m b \mid t b_{r}\right) \\
& \quad+M_{r}\left(t b_{r}-k b \mid t b_{r}\right) \mathrm{E}\left[v_{r}\left(t b_{r}\right) v_{r}^{\mathrm{T}}\left(t b_{r}\right)\right] M_{r}^{\mathrm{T}}\left(t b_{r}-m b \mid t b_{r}\right)
\end{aligned}
$$

where

$$
\begin{gathered}
\mathrm{E}\left[w\left(t b_{r}-k b\right) \underline{\tilde{x}}_{r}^{\mathrm{T}}\left(t b_{r} \mid t b_{r}-b_{r}\right)\right] \\
=\mathrm{E}\left[w\left(t b_{r}-k b\right) x^{\mathrm{T}}\left(t b_{r}\right)\right]=\mathrm{E}\left[\sum_{p=1}^{k} w\left(t b_{r}-k b\right) w^{\mathrm{T}}\left(t b_{r}-p b\right) \Gamma \Phi^{p-1}\right]=R^{w} \Gamma^{\mathrm{T}}\left(\Phi^{k-1}\right)^{\mathrm{T}}
\end{gathered}
$$

In (32), we use the fact that $\underline{\tilde{x}}_{r}\left(t b_{r} \mid t b_{r}-b_{r}\right)=x\left(t b_{r}\right)-\underline{\hat{x}}_{r}\left(t b_{r} \mid t b_{r}-b_{r}\right)$ and $w\left(t b_{r}-k b\right) \perp \underline{\hat{x}}_{r}\left(t b_{r} \mid t b_{r}-b_{r}\right)$. Substituting (32) into (31), we have (26). Using $\underline{\tilde{x}}_{r}\left(t b_{r} \mid t b_{r}\right) \perp \hat{w}_{r}\left(t b_{r}-k b \mid t b_{r}\right)$, we have

$$
\begin{gathered}
P_{r}^{x w}\left(t b_{r}, t b_{r}-k b \mid t b_{r}\right)=\mathrm{E}\left[\underline{\tilde{x}}_{r}\left(t b_{r} \mid t b_{r}\right) \tilde{w}_{r}^{\mathrm{T}}\left(t b_{r}-k b \mid t b_{r}\right)\right] \\
=\mathrm{E}\left[\underline{\tilde{x}}_{r}\left(t b_{r} \mid t b_{r}\right)\left(w\left(t b_{r}-k b\right)-\hat{w}_{r}\left(t b_{r}-k b \mid t b_{r}\right)\right)^{\mathrm{T}}\right]=\mathrm{E}\left[\underline{\tilde{x}}_{r}\left(t b_{r} \mid t b_{r}\right) w^{\mathrm{T}}\left(t b_{r}-k b\right)\right]
\end{gathered}
$$

From (7) and (23), we have the following filtering error equation

$$
\begin{gathered}
\underline{\tilde{x}}_{r}\left(t b_{r} \mid t b_{r}\right)=x\left(t b_{r}\right)-\underline{\hat{x}}_{r}\left(t b_{r} \mid t b_{r}\right)=\underline{\tilde{x}}_{r}\left(t b_{r} \mid t b_{r}-b_{r}\right)-K_{r}\left(t b_{r}\right) \underline{\varepsilon}_{r}\left(t b_{r}\right) \\
=\left[I_{n}-K_{r}\left(t b_{r}\right) H_{r}\right] \underline{\tilde{x}}_{r}\left(t b_{r} \mid t b_{r}-b_{r}\right)-K_{r}\left(t b_{r}\right) v_{r}\left(t b_{r}\right)
\end{gathered}
$$

Substituting (34) into (33), and using the Assumption 1, we have

$$
P_{r}^{x w}\left(t b_{r}, t b_{r}-k b \mid t b_{r}\right)=\left[I_{n}-K_{r}\left(t b_{r}\right) H_{r}\right] \mathrm{E}\left[\underline{\tilde{x}}_{r}\left(t b_{r} \mid t b_{r}-b_{r}\right) w^{\mathrm{T}}\left(t b_{r}-k b\right)\right]
$$

Using (32), we can obtain (27). Substituting (31) and (33) into (29), we can obtain (25).

Next, we shall give the following suboptimal CIF filter $\hat{x}_{o}(t b), l \in\left\{1, \cdots, c_{r}\right\}$ by using the well known CIF algorithm[6]

$$
\hat{x}_{o}(t b)=\sum_{r=1}^{Q} A_{r}(t b) \hat{x}_{r}\left(t b_{r}-l b \mid t b_{r}\right)
$$

where LFs $\hat{x}_{r}\left(t b_{r}-l b \mid t b_{r}\right), r \in\{1, \cdots, Q\}$ are computed by Theorem 1. The corresponding fusion weights $A_{r}(t b)$ are computed by

$$
A_{r}(t b)=\omega_{r}(t b)\left(\sum_{r=1}^{Q} \omega_{r}(t b) P_{r}^{-1}\left(t b_{r}-l b \mid t b_{r}\right)\right)^{-1} P_{r}^{-1}\left(t b_{r}-l b \mid t b_{r}\right)
$$

where $\omega_{r}(t b)$ are computed by 


$$
\omega_{r}(t b)=\operatorname{tr}\left(P_{r}^{-1}\left(t b_{r}-l b \mid t b_{r}\right)\right) / \sum_{r=1}^{Q} \operatorname{tr}\left(P_{r}^{-1}\left(t b_{r}-l b \mid t b_{r}\right)\right), \quad 0 \leq \omega_{r}(t b) \leq 1, \sum_{r=1}^{Q} \omega_{r}(t b)=1
$$

where $P_{r}\left(t b_{r}-l b \mid t b_{r}\right)$ are given by Theorem 2 .

Remark 2 From (36)-(38), we know that the proposed distributed fusion filter is suboptimal since the correlation between any two LFs is ignored. On the other hand, the computational cost is reduced since the computation of the cross-covariance matrices between any two sensor subsystems is avoided.

\section{Simulation Results}

Consider the following tracking system with three sensors

$$
\begin{aligned}
& x(t b+b)=\left[\begin{array}{ll}
1 & b \\
0 & 1
\end{array}\right] x(t b)+\sqrt{10}\left[\begin{array}{l}
{\left[\begin{array}{l}
b^{2} \\
2 \\
b
\end{array}\right] w(t b)} \\
b
\end{array}\right] \\
& z_{r}\left(t b_{r}\right)=H_{r} x\left(t b_{r}\right)+v_{r}\left(t b_{r}\right), \quad r=1,2,3
\end{aligned}
$$

where $x(t b)=\left[\begin{array}{ll}s(t b) & \dot{s}(t b)\end{array}\right]^{\top}, s(t b)$ and $\dot{s}(t b)$ are the position and velocity at $t b$ time instant. $b$ is the state update period. Our aim is to find the distributed suboptimal CIF filter $\hat{x}_{o}(t b)$.
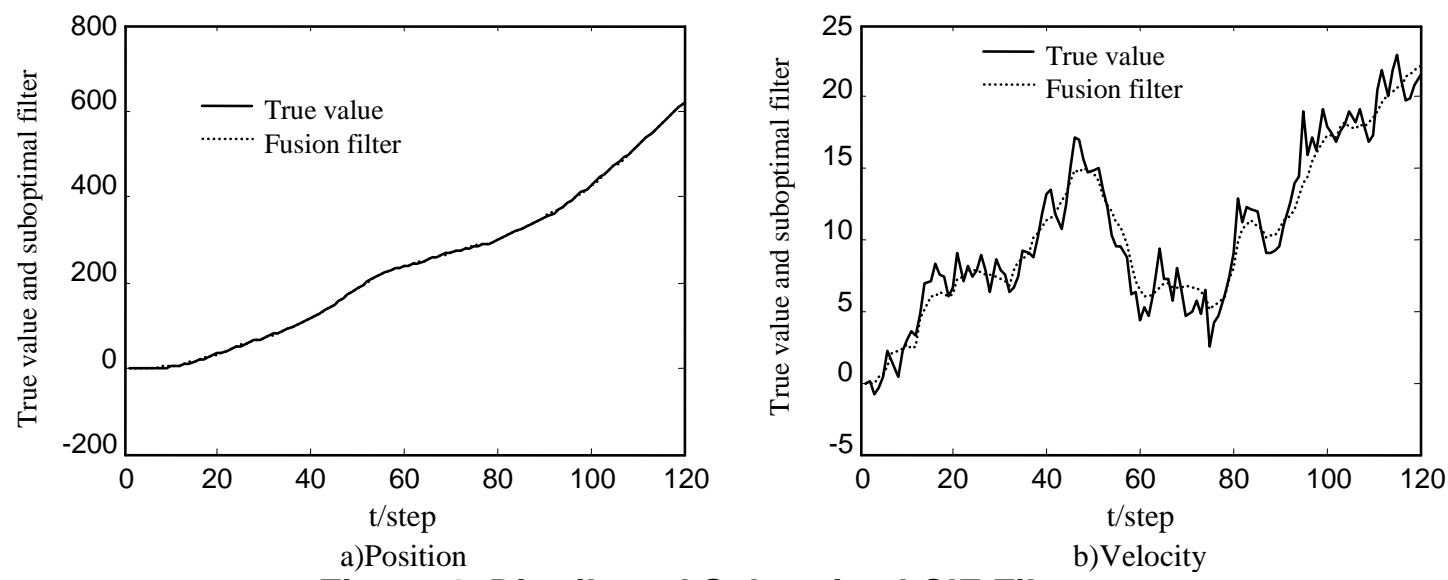

Figure 1. Distributed Suboptimal CIF Filter

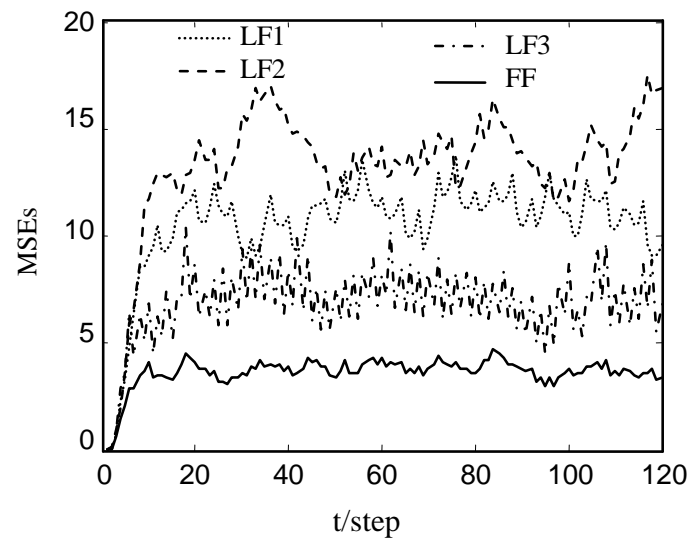

a)Position

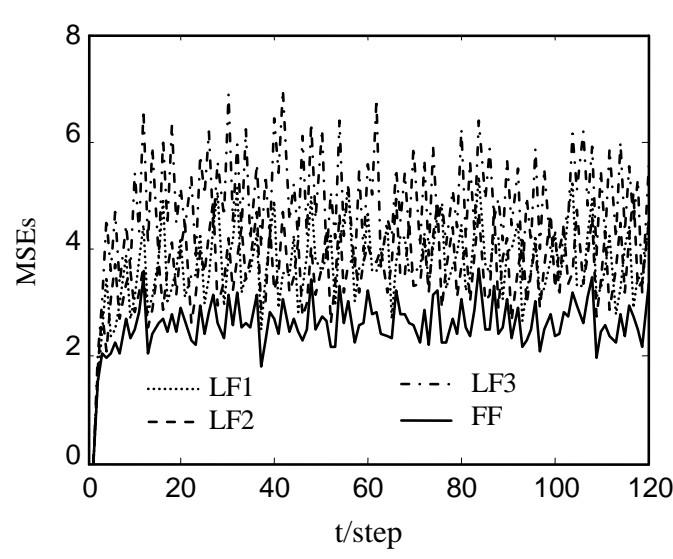

b) Velocity

Figure 2. Comparison Curves of MSEs of LFs and the CIF Filter 


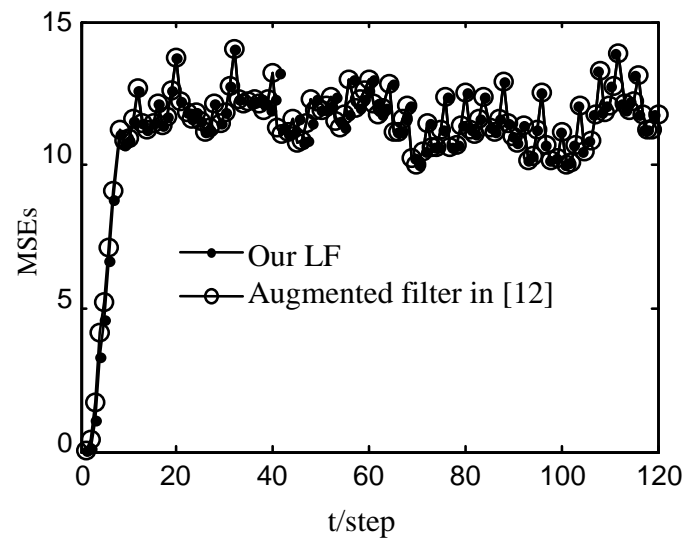

a)Position

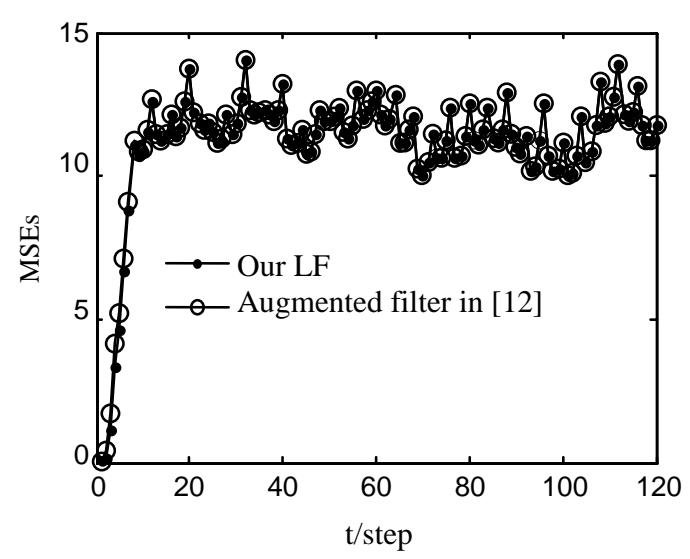

b)Velocity

Figure 3. Comparison Curves of MSEs of our LF and the Augmented Filter

In the simulation, we take 120 data and set $b=0.5$ second, $c_{1}=4, c_{2}=3, c_{3}=2$, $H_{1}=\left[\begin{array}{ll}1 & 1\end{array}\right], H_{2}=\left[\begin{array}{ll}0.5 & 1\end{array}\right], H_{3}=\left[\begin{array}{ll}1 & 0\end{array}\right], R^{w}=1, R_{1}^{v}=30 \quad, \quad R_{2}^{v}=15 \quad, \quad R_{3}^{v}=20 \quad$, $x(0)=\left[\begin{array}{ll}0 & 0\end{array}\right]^{\mathrm{T}}$ and $P_{0}=0.1 I_{2}$.

By using Lemma 1, we can obtain the LFs $\underline{\hat{x}}_{r}\left(t b_{r} \mid t b_{r}\right)$ and the corresponding filtering error variance matrices $\underline{P}_{r}\left(t b_{r} \mid t b_{r}\right)$ at the measurement sampling points. Further, the LFs $\hat{x}_{r}\left(t b_{r}-l b \mid t b_{r}\right), l=1,2, \cdots, c$, at state updating points can be obtained by using Theorem 1. The corresponding error variance matrices are given by Theorem 2 . Then, we can obtain the suboptimal CIF filter $\hat{x}_{o}(t b)$ by using (36)-(38).

The state tracking curves are given in Figure 1. The comparison curves of the mean square error (MSE) of the CIF and all LFs by 200 times Monte Carlo tests are given in Figure 2. From Figure 2, we see that proposed CIF has the higher accuracy than any LFs. In order to compare the computational cost and the accuracy of proposed LF and the filter in [12], we give comparison curves of the two filters for the first sensor subsystem. At the measurement sampling points, the computational cost of our LF is $\mathrm{O}\left(n^{3}\right)=\mathrm{O}\left(2^{3}\right)=\mathrm{O}(8)$, the computational cost of the filter in [12] is $\mathrm{O}\left(\left(c_{1} n\right)^{3}\right)=\mathrm{O}\left(8^{3}\right)=\mathrm{O}(512),\left(n=2, c_{1}=4\right)$. We see that our filter can obviously reduce the computational cost. The comparison curves of MSE by 200 times Monte Carlo tests are given in Figure 3. From Figure 3, we see that the two filters have the same estimation accuracy.

\section{Conclusion}

In this paper a multi-rate multi-sensor distributed suboptimal information fusion filtering problem for linear stochastic system is studied. Firstly, the LFs at the measurement sampling points are given by the classical Kalman filter. Then, the LFs at the state updating points are derived by the LFs at the measurement sampling points. The corresponding filtering error variance matrices are derived to obtain the fusion weights. Furthermore, a CIF algorithm is applied to fuse all the LFs. Simulation results show better performance than any LFs.

\section{Acknowledgements}

This work was supported by Foundation of Heilongjiang Education Committee (No. 12541632). 


\section{References}

[1] Q. Pan, Z. F. Wang, Y. Liang, F. Yang and Z. G. Liu, "Basic Methods and Progress of Information Fusion (II)", Control Theory \& Applications, vol. 29, no. 10, (2012), pp. 1233-1244.

[2] B. D. O. Anderson and J. B. Moore, "Optimal Filtering", Englewood Cliffs, New Jersey: Prentice-Hall, (1979).

[3] N. A. Carlson, "Federated Square Root Filter for Decentralized Parallel Processes", IEEE Trans. on Aerospace and Electronic Systems, vol. 26, no. 3, (1990), pp. 517-525.

[4] K. H. Kim, "Development of Track to Track Fusion Algorithm", Proceedings of American Control Conference, (1994); Maryland.

[5] S. L. Sun and Z. L. Deng, "Multi-sensor Optimal Information Fusion Kalman Filter", Automatica, vol. 40, no. 6, (2004), pp. 1017-1023.

[6] L. J. Chen, P. O. Arambel and R. K. Mehra, "Estimation Under Unknown Correlation: Covariance Intersection Revisited", IEEE Trans. on Automatic Control, vol. 47, no. 11, (2002), pp. 1879-1882.

[7] F. Ding and T. W. Chen, "Modeling and Identification of Multirate Systems", Acta Automatica Sinica, vol. 31, no. 1, (2005), pp. 105-122.

[8] B. S. Chen, C. W. Lin and Y. L. Chen, "Optimal Signal Reconstruction in Noisy Filter Bank Systems: Multirate Kalman Synthesis Filtering Approach", IEEE Trans. on Signal Processing, vol. 43, no. 11, (1995), pp. 2496-2504.

[9] J. Wang, C. Z. Han and X. R. Li, “Asynchronous Multisensor Data FusionX”, Control And Decision, vol. 17, no. 6, (2001), pp. 877-881.

[10] Q. B. Ge, G. A. Wang, T. H. Tang and C. L. Wen, "The Research on Asynchronous Data Fusion Algorithm Based on Sampling of Rational Number Times", Acta Electronica Sinica, vol. 34, no. 3, (2006), pp. 544-548.

[11] A. B. Qiu, C. L. Wen and B. Jiang, "Optimal State Fusion Estimate Based on Asynchronous Multisensor Sampled Measurements”, Acta Electronica Sinica, vol. 38, no. 7, (2010), pp. 1483-1488.

[12] Y. Liang, T. W. Chen and Q. Pan, "Multi-rate Optimal State Estimation", International Journal of Control, vol. 82, no. 11, (2009), pp. 2059-2076.

[13] J. Ma, H. L. Lin and S. L. Sun, "Distributed Fusion Filter for Asynchronous Multi-rate Multi-sensor Non-uniform Sampling Systems", Proceedings of the 15th International Conference on Information Fusion, (2012); Singapore.

[14] Y. L. Liu, L. P. Yan, Y. Q. Xia, M. Y. Fu and B. Xiao, "Multirate Multisensor Distributed Data Fusion Algorithm for State Estimation with Cross-Correlated Noises", Proceedings of the 32nd Chinese Control Conference, (2013); Xi'an.

[15] F. F. Peng and S. L. Sun, "Distributed Fusion Estimation for Multi-sensor Multi-rate Systems with Stochastic Observation Multiplicative Noises", Mathematical Problems in Engineering, Article ID 373270, (2014).

[16] J. Ma and S. L. Sun, "Distributed Fusion Filter for Multi-rate Multi-sensor Systems with Packet Dropouts", Proceedings of the 10th World Congress on Intelligent Control and Automation, (2012); Beijing

[17] M. S. Mahmoud and M. F. Emzir, "State Estimation with Asynchronous Multi-rate Multi-smart Sensors", Information Sciences, vol. 196, (2012), pp. 15-27.

[18] W. A. Zhang, G. Feng and L. Yu, "Multi-rate Distributed Fusion Estimation for Sensor Networks with Packet Losses", Automatica, vol. 48, no. 9, (2012), pp. 2016-2028.

\section{Authors}

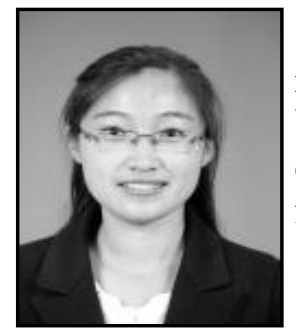

Jing Ma, she was born in Heilongjiang, China, in 1979. She received the M.E. and Ph.D. degrees from Heilongjiang University, Harbin, China, in 2007 and 2012, respectively. Her current research interests include sensor network and information fusion filtering. 


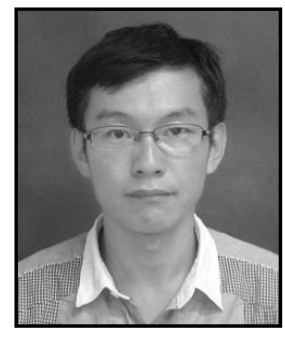

Hao Jin, he was born in Heilongjiang, China, in 1981. He received the B.E. and M.E. degrees from Heilongjiang University, Harbin, China, in 2004 and 2007, respectively. His current research interests include information fusion filtering and image processing.

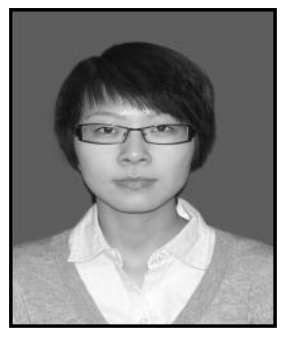

Tian Tian, she was born in China, in 1981. She received the B.E. degree from Jilin University of Technology, China, in 2004, and M.E. degree from Heilongjiang University, China, in 2010. Her research interests include state estimation and time delay systems.

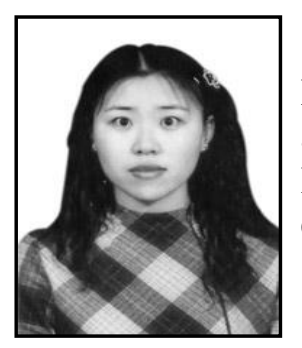

Wei Zhang, she was born in China, in 1979. She received the B.E. degree from Mudanjiang normal university, China, in 2001 and M.E. degree from Heilongjiang University, China, in 2007. Her current research interest is numerical solution of differential equations. 
International Journal of Multimedia and Ubiquitous Engineering

Vol. 10, No. 1 (2015) 